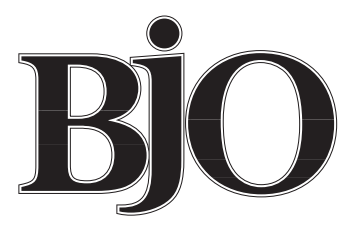

British Journal of Ophthalmology

Recent estimates suggest that nearly 45 million people worldwide fulfil the World Health Organization's criterion for blindness, defined as a best corrected vision of less than 3/60 in the better seeing eye. ${ }^{1}$ An additional 135 million people are visually disabled and in need of social, vocational, economic, or rehabilitative support services. To compound matters, more than $90 \%$ of all blind and visually disabled people live in the developing world, where common causes of bilateral vision loss include cataract, glaucoma, trachoma, vitamin A deficiency, and onchocerciasis. Additional causes of bilateral vision loss, which together comprise nearly one quarter of all blindness and which affect people in both developed and developing nations, include diabetic retinopathy and macular degeneration, among others (Fig 1). These numbers are projected to double within two to three decades unless innovative blindness prevention initiatives are undertaken in the near future.

It is against this backdrop that the editors of the $B \mathcal{O} O$ have elected to introduce a new column entitled "World view," which will address issues pertaining to blindness prevention in the broadest sense. Hugh Taylor and Jill Keeffe provide the inaugural instalment for "World view" with their essay entitled "World blindness: a 21 st century perspective" (p 261). Together, Taylor and Keeffe highlight some of the past successes in blindness prevention, including vaccination for smallpox and the

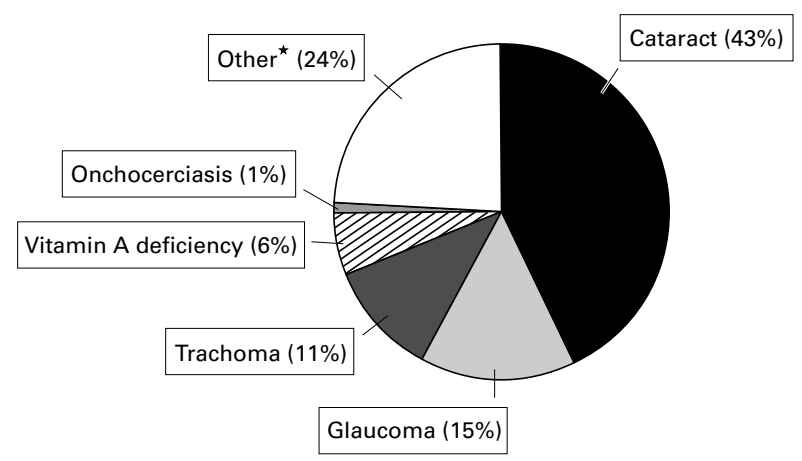

${ }^{\star}$ Diabetic retinopathy, macular degeneration, optic neuropathy, etc

Figure 1 The World Health Report, 1998. ${ }^{1}$ Worldwide causes of blindness for 1997. Total number of blind (vision <3/60) estimated at 44800000. introduction of Crede prophylaxis for the prevention of ophthalmia neonatorum in the 19 th century, and the use of insulin to treat diabetes mellitus and the development of modern cataract surgery in the 20th century. Taylor and Keeffe caution, however, that the 21 st century will present new, and possibly even greater, hurdles. Many people in developing nations still have no access to well established blindness prevention measures despite their long standing record of effectiveness in Europe and North America. In addition, the global population is both growing and ageing at a rapid pace. This means that both the prevalence of blindness and the absolute number of people with profound vision loss will increase dramatically, particularly vision loss due to cataract and other age related disorders, such as diabetic retinopathy and macular degeneration. Lastly, but most importantly, is the ever increasing realisation that functional blindness occurs long before vision drops to 3/60. In fact, Taylor and Keeffe suggest that "economic blindness" probably occurs once vision drops below $6 / 12$, since vision below this level often affect a person's ability to drive and to function effectively in the workplace. Resetting this benchmark for functionally significant vision loss will, perhaps more than any other factor, magnify the global burden of blindness in years to come.

While world blindness may, at first glance, seem like an intractable problem, and to be sure many challenges lie ahead, a number of talented and dedicated vision researchers around the world are hard at work on blindness prevention. Many successes have already been realised, and the future, we believe, holds great promise. Yet, despite its undeniable importance, "World view" is the first column in an ophthalmology journal to be dedicated entirely to issues of world blindness. In some ways, "World view" is a work in progress. Feedback from our readers will be invaluable, therefore, to help shape this column as it evolves over the coming months. We look forward to your helpful comments and suggestions.

This work was supported in part by a career development award from Research to Prevent Blindness, Inc, New York, NY, USA.

EMMETT T CUNNINGHAM, JR

The Pearl and Samuel J Kimura Ocular Immunology Laboratory, the Francis I Proctor Foundation, and the Department of Ophthalmology, UCSF Medical Center, San Francisco, CA 94143-0944, USA

emmett@itsa.ucsf.edu

1 World Health Organization. Programme for the prevention of blindness and deafness. Global initiative for the elimination of avoidable blindness. (WHO/PBL/97.61). Geneva: WHO, 1998:1-2. 


\section{Cataract surgery in young children}

Every minute of the day a child is born blind or acquires blindness, with most cases of blindness occurring in developing nations. ${ }^{1}$ The excess in prevalence of childhood blindness in the poorest regions of the world is caused by many factors. Vitamin A deficiency and measles lead to corneal scarring; inadequate vaccination programmes result in high rates of congenital rubella and acquired rubeola; and inadequate treatment programmes allow manageable disorders such as congenital glaucoma and retinopathy of prematurity to damage vision. Many of these causes of blindness could be avoided or treated with proper resources.

One of the most common and avoidable causes of blindness in children in the developing world is cataract. ${ }^{2}$ Experience of treating childhood cataract in the United States is illuminating in this regard. Cataract ranked as a leading cause of childhood blindness in the USA 40 years ago, ${ }^{3}$ but with modern surgical techniques, improved diagnostic programmes, and rubella vaccination programmes cataract is now an uncommon cause of childhood blindness. Unfortunately, superimposing successful cataract management programmes from well to do regions onto poor regions is not so simple. The task of surgical and postoperative management of aphakia is particularly daunting. Glasses are easily lost and contact lens use is virtually impossible, leaving aphakic children with blurred vision during a critical period of visual development.

In this month's issue of the BFO (p 267), Yorston and colleagues report their experience using intraocular lenses for childhood cataract in east Africa, where cataracts rank as the leading cause of paediatric blindness. The majority of children $(76 \%)$ were blind preceding surgery, and $42 \%$ had "sensory" nystagmus. With implantation of an intraocular lens (IOL) at the time of surgery, $44 \%$ had visual acuity of $6 / 18$ or better and $91 \%$ had corrected acuity of $6 / 60$ or better. The authors had success with several types of IOL and with capsular or sulcus fixation. The results of this study allow new optimism for those who manage paediatric cataract in poor regions of the world. For those practising in developed regions, there are important and cautionary lessons as well.

The first note of caution is sounded by the occurrence of severe fibrinous uveitis in $30 \%$ of cases, with sulcus or capsular placement of the IOL. In five children this resulted in a fibrinous membrane that required further surgery. Distorted pupils also occurred in a few children and may have been caused by uveitis. Since follow up in this study was short (3 months in most cases), we do not know the long term ramifications of severe uveitis. In a developing region, uveitis seems a reasonable risk when no aphakia management option other than an IOL is available. In a developed region where contact lenses and glasses are an option, an incidence of $30 \%$ is alarming.

A second lesson comes from the group's experience with amblyopia. Children operated before the age of 2 were more likely to have amblyopia, presumably because their cataracts were visually significant during a critical period of visual development. Esotropia was also more likely to occur in the younger children with cataracts. Successful surgical management loses much of its significance if amblyopia is not managed properly. It is not enough to provide expert surgical care to young children. An understanding of the diagnosis and management of amblyopia is equally important.

Biometry was an important aid to surgery in this study. When used, it nearly halved the postoperative refractive error, on average. Most patients were left with hyperopia, which showed a trend towards emmetropisation with time. But if replacement of glasses and contact lenses is a priority in developing regions, the argument could be made that amblyopia would be better avoided if the patient were left emmetropic or even slightly myopic. Then the child would have a usable focal distance, thereby ensuring better visual development without correction.

Intraoperative management often included removal of the posterior capsule and sulcus placement of the IOL, in this study. Postoperative management of the posterior capsule is problematic for a number of reasons in any region of the world: the young, awake child's posterior capsule may pose a formidable laser target; posterior capsule opacification often includes thickening, requiring higher laser energy; and posterior capsule scarring may recur, necessitating additional treatment.

The authors' experience teaches us an important lesson in neurophysiology. In most children where nystagmus had developed as a result of cataracts, it disappeared after treatment. The developing ocular motor system acquires nystagmus as the result of inadequate afferent input in early childhood, but well timed removal of the sensory defect evidently allows recovery and steady visual fixation. In regions where direct ophthalmoscopy is unavailable, or when cataracts are developmental and not easily identified, nystagmus might be a useful marker of ocular defects. Prompt correction of the defect will promote ocular motor stability, a sign of vision rehabilitation.

The future of any society is its children. The renewed goals of the World Health Organization (WHO) to improve the care of children with blindness and vision impairment is especially prescient, since childhood blindness almost equals adult blindness when measured in blind years of life. ${ }^{1}$ Childhood blindness significantly shortens life expectancy and imposes an enormous economic, psychological, and social hardship on its victims. The results reported in this month's issue of the $B F O$ truly have global implications for children. For those caring for children in underdeveloped regions where glasses and contact lenses are not available, IOLs seem a reasonable option, but an option not without risk. And for those caring for children in developed regions, surgeons should carefully consider the side effects of IOLs reported in this study.

Supported by NEI grant 00384 .

WILLIAM V GOOD

Smith-Kettlewell Eye Research Institute, 2318 Filmore Street, San Francisco, CA 94115, USA

Good@Ski.org

1 Global initiative for the elimination of avoidable blindness. WHO Bull 1997;97:17-21.

2 Waddell KM. Childhood blindness and low vision in Uganda. Eye 1998;12: 184-92.

$3 \mathrm{Kahn} \mathrm{H}$, Moorhead H. Statistics on blindness in the model reporting area, 1960-1970. Public Health No NIH 73-427. Washington, DC: US Department of Public Health, 1973. 


\section{Gene therapy in hereditary retinal degeneration and the tower of Babel}

In the Western world hereditary retinal diseases are the most common cause of blindness in people under 70 years of age, affecting about 1.5 million individuals. Various attempts have been tried but none of the enumerated treatments had any scientifically confirmed beneficial effect. ${ }^{1}$ Gene therapy holds the promise of revolutionising the treatment of genetic diseases and might be an "ideal" approach to treat many forms of hereditary retinal diseases. Indeed, hereditary retinal diseases meet all of the major requirements for gene therapy. ${ }^{2}$ Firstly, their genetic basis is well characterised and the biochemical defects are known in several diseases (for example, Refsum disease, gyrate atrophy, Kearns-Sayre syndrome). Secondly, efficient gene delivery techniques that can be relatively well controlled are available and allow even local ocular application. Lastly, reliable animal models of hereditary retinal diseases are available that permit preclinical testing.

However, fundamental challenges in gene therapy are still present and it appears that clinical trials in non-life threatening disorders such as retinal dystrophies are far away from being conducted. One of the most significant hurdles preventing the clinical application of gene therapy is the lack of safe gene transfer systems. Of all gene delivery vectors available today, viral vectors, including replication defective adenoviruses, adeno associated viruses, herpes simplex 1, and lentiviruses, dominate the field. Replication defective adenoviral vehicles are probably the most versatile vectors to deliver plasmid DNA to retinal cells. Most importantly, at low multiplicity of infection they do not appear to interfere with RPE cell function or survival. ${ }^{3}$ However, adenoviral vectors remain episomal and cannot integrate into the host genome for long term gene expression. In addition, first generation E1 deleted adenoviruses express proteins of their own, resulting in an immune response against the cells that harbour the virus and lead to clearance of the infected cells with ultimately loss of the therapeutic effect. Indeed, a number of studies are showing a shortened term of expression due to a potent antiviral immune response. ${ }^{4}$

The paper presented by Reichel et al (p 341) in this issue of the $B F O$ reports interesting observations, which in part contradict these concerns, and contributes to the growing literature on unexpected findings in ocular gene therapy. Following intravitreal injection of an adenoviral vector carrying a reporter gene, a significant rescue of photoreceptors was observed when injections were performed in $\mathrm{T}$ cell depleted $r d$ mice. Although previous studies already reported that ocular injury, such as intravitreal injection itself, can inhibit photoreceptor cell degeneration, it is not considered as a significant factor. The results presented in this study indicate that the immune response may have a so far unexpected role following adenovirus mediated gene transfer. The authors substantiate their findings by experiments using depletion of either CD4+, CD8+ cells or a combined strategy that support the finding of an immune mediated protective effect. It is of interest that depletion of both CD4+ and CD8+ cells was necessary to obtain protection indicating that the afferent as well as the efferent arc of the immune response is involved.

Still the central question remains, how is this mechanisms generated? The authors hypothesise a role of growth factors and this is certainly a vivid explanation. There is growing evidence that neuroprotective factors such as basic fibroblast growth factor (bFGF) and ciliary neurotrophic factor (CNTF) are upregulated following injury. ${ }^{5}$ However, the finding that animals receiving sham injections did not demonstrate a beneficial effect strongly suggests that this is not yet an unspecific bystander effect. Instead, activated $\mathrm{T}$ cells seem to participate in retina protection. Interestingly, this may highlight another field of interest that recently has been coined as "protective autoimmunity". ${ }^{6}$ Reactive $\mathrm{T}$ cells, usually considered as the "bad guys", have been shown to have a protective role in neurodegeneration. Activated $\mathrm{T}$ cells are known to patrol the central nervous system including the neuroretina and an immune response may act as a protective mechanism. ${ }^{6}$

Another question that remains open is the relative immunogenicity of viral antigens compared with the transgene. It might be assumed that expression of the encoded proteins following transfection is more immunogenic than the intracellular reporter gene product, but no proof is provided. Since gene therapy for hereditary retinal diseases is an active field of interest, this has to be kept in mind and further investigations seem indicated. In particular the use of adequate controls including an inactive "functional" gene or immunosuppressive treatment seems mandatory and has also been suggested by the authors of this study.

Taken together, the findings of this report may at least bring to mind that more basic research is needed in gene therapy and no rush into clinical trials should be expected. This resumé already given 5 years ago still holds true. ${ }^{7}$ In the short term it seems more likely that advances in the treatment of hereditary retinal degenerations may come from other therapeutic options-for example, retinal implants. ${ }^{1}$ However, the ultimate goal of a definitive permanent treatment lies in the future of gene therapy.

U PLEYER

University Eye Hospital, Charite/Virchow Hospital, Humbolt

University, Augustenburger Platz 1, 13353 Berlin, Germany

1 Sharma RK, Ehinger B. Management of hereditary retinal degenerations: present status and future directions. Surv Ophthalmol 1999;43:427-44. 2 Mulligan RC. The basic science of gene therapy. Science 1993;260:926-32.

3 Bennett J, Tanabe T, Sun D, et al. Photoreceptor cell rescue in retinal degeneration (rd) mice by in vivo gene therapy. Nat Med 1996;2:649-54.

4 Reichel MB, Ali RR, Thrasher AJ, et al. Immune responses limit adenoviral mediated gene expression in the adult mouse eye. Gene Ther 1998;5:103846.

5 Wen R, Song Y, Cheng T, et al. Injury-induced upregulation of bFGF and CNTF mRNAS in the rat retina. $\mathcal{F}$ Neurosci $1995 ; 15: 7377-85$.

6 Moalem G, Leibowitz-Amit R, Yoles E, et al. Autoimmune T cells protect neurons from secondary degeneration after central nervous system axotomy. Nat Med 1999;5:49-55.

7 Marshall E . Less hype, more biology needed for gene therapy. Science 1995; 270:1751. 


\section{Commentaries}

The $B F O$ is giving a new look to its commentaries. There will be a number of styles of commentaries, either by invited authors or as submitted articles: all will be peer reviewed. They will deal with a wide variety of topics, not necessarily related directly to papers in the current or a recent issue. Although many will comment on the current science of our specialty, some will have a style reminiscent of a newspaper commentary, sometimes being about broadly ethical or political topics. They will be factual and topical, but may include opinion; most will be masterpieces of compression rather than being exhaustive and fully referenced. With luck, some will be amusing or witty, at least in parts, but they will all aim to be practical and helpful to ophthalmologists and visual scientists of diverse backgrounds. Although many of the commentaries will be by invited authors, we hope for submissions from anyone involved in vision science in the broadest sense, and at any stage in their career.

DAVID TAYLOR

Eye Department, Great Ormond Street Hospital, London WC1N 3JH, UK

\section{Video Reports (www.bjophthalmol.com)}

Capsule staining and mature cataracts: a comparison of indocyanine green and trypan blue dyes. D F Chang

Pearls for implanting the Staar toric IOL. D F Chang 